\title{
ON THE CALCULATION OF BULK PROPERTIES OF HETEROGENEOUS MATERIALS*
}

\author{
BY \\ JOHN J. MCCOY \\ Catholic University, Washington, D. C.
}

\begin{abstract}
Models for calculating the effective, or bulk, properties of heterogeneous materials are considered in the light of a Dyson equation formalism. It is seen that convergence difficulties, of the same nature as those which necessitated the renormalization effected by the Dyson equation in the first place, are frequently reintroduced, but now in calculating the parameters to use in the equation. Thus the validity of the models must be suspect. Fortunately, in most instances the validity of the models, if properly interpreted, can be established. The proper interpretation to be given is considered.
\end{abstract}

Introduction. In the more common definitions of effective, or bulk, property measures one assumes a statistically homogeneous test specimen forced so as to result in statistically homogeneous flux (e.g. charge, heat, momentum) and gradient (e.g. potential, temperature, displacement) fields [1]. The effective property measures are defined as quantities that give the correct proportionality between the (ensemble) averaged, and hence constant, flux and gradient fields [2]. For test specimens forced so as to result in flux and gradient fields with statistics that might be termed quasi-homogeneous, an assumption is made that the averaged flux and gradient fields, no longer constants, are equal to fields calculated for a fictitious, effective medium. That is, one assumes that the averaged field response is properly given by an "effective modulus theory." The condition of quasihomogeneity can be made more precise by a statement that the length scale for variations in the averaged fields (i.e. the problem macroscale) is to be large compared to length scales for measuring the random variations (i.e. the problem microscale).

An "effective modulus theory" can be considered to be derived by a general formalism that needs to be satisfied by the averaged inverse of a linear stochastic operator. We shall term this general formalism a Dyson equation formalism [3], and note that it reduces to the effective modulus theory for the two-length-scale situation intuitively introduced above. The Dyson equation formalism provides another definition of effective property measures. One of the purposes of the present paper is to consider the relationship between these seemingly different definitions of effective property measures.

The Dyson equation formalism can be obtained by two procedures, one frequently termed the method of smoothing and one termed a renormalization. The motivations prompting the two procedures are distinctly different, although the end product is the same. In the context of the heterogeneous media problem, the method of smoothing

\footnotetext{
* Received March 23, 1978; revised version received July 17, 1978. The study reported herein is an outgrowth of numerous discussions, held during a sabbatical leave visit, with both the permanent staff of and visitors to the Department of Applied Mathematics and Theoretical Physics, Cambridge University. The study was supported by the National Science Foundation under grant number ENG 76-11549.
} 
appears to incorporate explicitly the two length scales of the problem and the idea that a microscale response is related to (determined by) a local macroscale response through the solution of a canonical problem. The derivation of the Dyson equation consists in a formal solution of the canonical problem, which is substituted into the averaged field equation to give the desired equation on the mean field, or macroscale response. The renormalization considers an iteration (e.g. perturbation) solution to the problem. Then, convergence difficulties of the iterated solution are noted in the appearance of conditionally convergent terms. To remove these convergence difficulties, the iterated solution is renormalized using the Dyson equation formalism.

In reducing the definition of an effective property measure as a ratio of an averaged, homogeneous flux field and an averaged, homogeneous gradient field to computational algorithms, one usually is required to look to an iterated solution. As might be expected, convergence difficulties of the same nature as those which necessitated the use of an effective modulus theory in the first place can arise in the prescriptions for the values of the effective modulus to use in the theory. Unfortunately, these difficulties are not always recognized as such, with the result that the prescriptions for the effective property measures can be in error if not interpreted properly. A second objective of the present paper is to expose this point.

The Dyson equation formalism appears first to have been applied to the bulk properties of heterogeneous materials by Beran and McCoy [4, 5]. The derivation used the method of smoothing and hence did not emphasize the fact that the result actually effected a renormalization. The formalism has been applied more recently by Zeller and Dederichs [6] and by Gubernatis and Krumhansl [7]. The derivations in these latter studies have much in common with classical scattering theory, although in neither paper are the convergence difficulties noted. The problem in which these difficulties were first explicitly noted dealt with the effective viscosity of a fluid suspension. While Batchelor and Green [8] credit Peterson and Fixman [9] with recognizing the difficulty early on, and with overcoming it in a correct fashion, it was actually through the efforts of Batchelor and his colleagues [10-13] that the nature of the difficulty became clear. These last authors' interests were not with the development of a general formalism but with the specific problem of calculating an effective property measure to order $C^{2}$ where $C$ is the concentration of the suspending particles. The importance of long-range interactions was clearly noted in these studies, as was the fact that these long-range interactions lead to convergence difficulties. The renormalization used to circumvent the difficulties was first developed by Batchelor [10] in the context of sedimentation. Although the last-referenced authors were clearly aware that the problem encountered was due to the slow rate of decay of a disturbance caused by an inclusion, they did not connect their work to the scattering theories in which even stronger convergence difficulties arise. Still a third objective of the present paper is to indicate the relationship between the studies by Batchelor and his colleagues with the studies based on the Dyson equation. A more detailed review paper in which this relationship is made more precise is being prepared by the author.

The Dyson equation. We consider the following operator equation:

$$
L u=f,
$$

where $L$ denotes a linear stochastic operator, $f$ denotes a forcing taken to be deterministic and $u$ is the solution field. From this the following equation can be derived for the ensemble averaged, i.e. the mean, solution field denoted by $\langle u\rangle$ (see Frisch [14], for example, for details):

$$
(\langle L\rangle-M)\langle u\rangle=f,
$$


where $M$ is a deterministic operator that can be represented by the infinite series

$$
M=\sum_{n=1}^{\infty}(-)^{n}\left\langle L_{1}\left[G_{0}(I-P) L_{1}\right]^{n}\right\rangle .
$$

An angular bracket indicates an ensemble average; $G_{0}$ denotes a Green's function operator for the averaged medium [15]; $P$ denotes the taking of an ensemble average; $I$ is the identity operator; and a subscript one indicates the fluctuating component of the indicated quantity taken about its mean value, i.e.,

$$
L_{1}=L-\langle L\rangle=(I-P) L .
$$

Eq. (2) is referred to as a Dyson equation, and the operator $M$ is termed either a mass operator or an effective index operator. The latter term is more descriptive of the problem of interest in this paper.

A "proof" of the Dyson equation formalism is provided by constructing the perturbation solutions of both Eqs. (1) and (2) (using the averaged operator $\langle L\rangle$ to provide the base solution), taking the average of the former, and noting that the two series are identical [16]. In carrying out this comparison it is convenient to introduce a diagrammatic notation. Since this notation is also an aid to understanding Eq. (3) and the ideas of interest in this paper, we introduce it here. The basic diagrammatic elements are a line segment - used to denote the $G_{0}$ operator; a small dot $\bullet$, used to denote the $\left(-L_{1}\right)$ operator; and a dashed line -- , used to connect combinations of $L_{1}$ operations to be joined in taking an average. With these elements the effective index operator, denoted diagrammatically by a large dot $O$, is given by

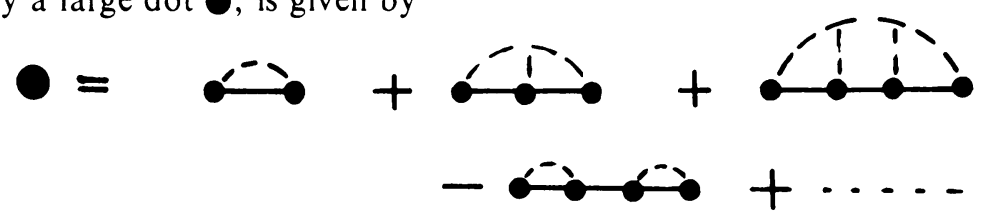

In Eq. (5) we have reproduced terms up to and including those of fourth order in the magnitude of $L_{1}$.

Diagrams have received a good deal of attention in the literature of the scattering of radiation fields, and borrowing some of the terminology of that field is instructive for interpreting Eq. (5). Like the physical scattering problem, in our problem, the $L$ and $L_{1}$ operators are in general differential operators whereas $G_{0}$ is an integral operator. Thus, one can interpret $L_{1}$ as a local operator describing the interaction of the random microstructure and the field on which $L_{1}$ operates. It is a scattering operator. On the other hand, $G_{0}$ can be interpreted as a nonlocal operator representing the dispersal of the effects of the local $L_{1}$ operations to other points in the medium. The $G_{0}$ operator is, in effect, a propagator. Each term in the series of Eq. (5) can be interpreted in terms of combinations of scatterings and propagations. In the first term we need consider two scatterings joined by a single propagation; in the second we need consider three scatterings joined by two intermediate propagations, etc. The averaging operator, represented in Eq. (5) by a dashed line, places restrictions on the combination of scatterings that one must incorporate. Thus, in considering the first term the two scatterings must be such as to be correlated. (We recall that $L_{1}$ has been chosen such that $\left\langle L_{1}\right\rangle=0$.) In a physical problem, this will restrict the scatterers to lie within a neighborhood of one another. The extent of the neighborhood is determined by a correlation length of the random microstructure. Similarly, in considering the second term of Eq. (5), all three scatterings must be such as to be correlated one to another. Thus, all three must lie within a neighborhood of one another. A difference is to 
be noted in considering the third term of Eq. (5) since there is no requirement that all four scatterings be correlated one to another for this term to provide a significant contribution. All that is required is that the scatterings correlate in pairs. To emphasize this fact it is common to introduce a cluster expansion, or an expansion in terms of cumulants, of this third diagram, and write it as

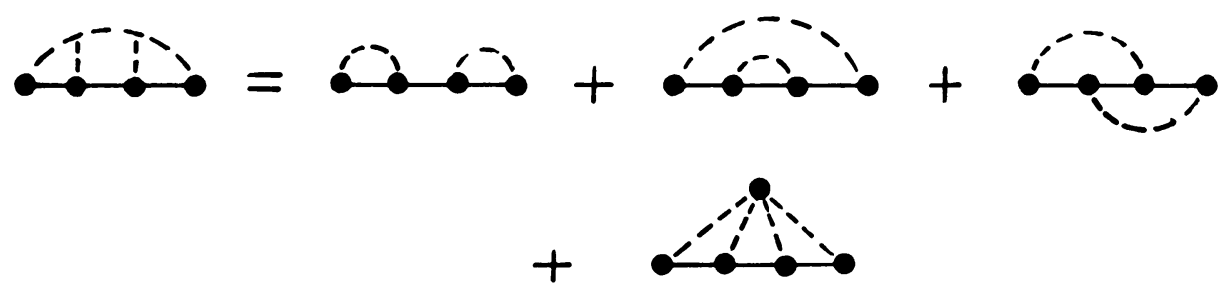

Actually, this equation serves to define the last term on the right-hand side. The purpose in writing it is to explicitly separate the pairwise clustering of scatterings that raise the possibility of long-range interaction. These pairwise clusterings are given by the first three terms. In comparing these three terms we notice that for the first a single propagation separates one clustered pair from the second, whereas for the second and third terms, two and three propagations separate the two cluster pairs, respectively. Since the effects of a propagation fall off with increasing range, it is to be expected that for far-separated cluster pairs the first term of the three will dominate, with the size of the contributions of the second and third following in order.

The importance of the long-range contribution of scatterings by scatterers that cluster in pairs (or triplets, etc.) depends entirely on the rate of fall-off of the propagator with range. For either the physical scattering experiment or the static problem of interest here, the fall-off is sufficiently slow that the first term of Eq. (6) leads to convergence difficulties. In the physical scattering experiment, the difficulties encountered are actual divergences; in the problem of interest here conditional convergences are encountered, which can lead to the physically unsatisfactory result that the bulk properties depend on the shape of the bulk specimen. We shall not consider the physical scattering experiment further: the conditional convergence encountered in our statical problem will become clear when the formalism is reduced to a specific problem. We further note here that the fall-off of the propagator with range for our problem is sufficiently rapid that the second and third terms of Eq. (6) do not contain the convergence difficulty of the first term.

If Eq. (6) is substituted in Eq. (5), it is seen that the fourth term of Eq. (5) subtracts out just that portion of the third term that leads to a lack of absolute convergence. The importance of the Dyson equation formalism to the bulk field problem is just that; it removes, or renormalizes, those terms of an iterated solution that lead to the conditional convergence difficulties isolated above [17]. As we shall see, however, the removal of the convergence difficulties that is accomplished by the Dyson equation formalism can be negated by an improperly-chosen prescription for evaluating the effective index operator.

In this paper we take the heat conduction problem as a representative of the many other effective index problems. The relationships between the several types of problems have been noted in many references $[18,19]$. We thus write the governing stochastic field equations as

$$
\nabla \cdot \mathbf{q}=\rho(\mathbf{x}),
$$

and

$$
\mathbf{q}=\epsilon(\mathbf{x}) \nabla T,
$$


where $\mathrm{q}(\mathbf{x})$ and $T(\mathbf{x})$ denote the heat flux field and the temperature field, respectively; $\rho(\mathbf{x})$ is a deterministic source term; and $\epsilon(\mathbf{x})$ is the local thermal conductivity. Local isotropy has been assumed. To keep the mathematics rigorous in accomplishing all intermediate calculations, we consider the fluctuations in $\epsilon(\mathbf{x})$ to be confined to a finite region outside which $\epsilon(\mathbf{x})$ is constant, equal to its averaged value [20]. No convergence difficulties are encountered for the Dyson equation formalism upon subsequently allowing this region to increase without bound.

The Dyson equation for Eqs. (7) and (8) can be written [4] (an integration by parts is required to obtain the formulation in this form)

$$
\begin{gathered}
\nabla \cdot\langle\mathbf{q}\rangle=\rho(\mathbf{x}) \\
\langle\mathbf{q}\rangle=\varepsilon_{N L}^{*} \cdot\langle\nabla T\rangle,
\end{gathered}
$$

where $\varepsilon_{N L}^{*}$ is a nonlocal effective thermal conductivity operator that can be written as

$$
\varepsilon_{N L}^{*}=\langle\varepsilon\rangle\left[1+\delta_{N L}^{*}\right],
$$

where 1 is the unit dyad, and $\boldsymbol{\delta}_{N L}^{*}$ is given diagrammatically by Eq. (5). The small dot is to be identified with the scalar operation

$$
\frac{\epsilon_{1}\left(\mathbf{x}^{\prime}\right)}{\langle\epsilon\rangle} \varphi\left(\mathbf{x}^{\prime}\right),
$$

and the line segment with the integral operation

$$
\int \mathbf{G}\left(\mathbf{x}, \mathbf{x}^{\prime}\right) \cdot \varphi\left(\mathbf{x}^{\prime}\right) d \mathbf{x}^{\prime},
$$

where $\varphi(\mathbf{x})$ is a generic vector field on which the operation is to be taken and $\mathbf{G}\left(\mathbf{x}, \mathbf{x}^{\prime}\right)$ is a dyadic field given by

$$
\mathbf{G}\left(\mathbf{x}, \mathbf{x}^{\prime}\right)=\nabla \nabla G\left(\mathbf{x}, \mathbf{x}^{\prime}\right)=\nabla \nabla\left(\frac{1}{4 \pi r}\right) .
$$

The scalar field $G\left(\mathbf{x}, \mathbf{x}^{\prime}\right)$ is the free-space Green's function for Laplace's equation, $r$ being the separation distance between the source and field points. The definition of $\mathbf{G}\left(\mathbf{x}, \mathbf{x}^{\prime}\right)$ requires the introduction of a distribution. Following Saffman [21] we can write it as

$$
\mathbf{G}\left(\mathbf{x}, \mathbf{x}^{\prime}\right)=\frac{3 \hat{\mathbf{r}} \hat{\mathbf{r}}-1}{r^{3}}-\frac{4 \pi}{3} \delta(\mathbf{r}) 1,
$$

where $\hat{r}$ is a unit vector. A $n$ integral over the singularity at $r=0$ for this first portion of Eq. (15) is to be understood in a Cauchy principal-value sense.

A local theory, which is valid if the length scales for variations in the mean field variables $\langle\mathbf{q}\rangle$ and $\langle\nabla T\rangle$ are large compared to the length scales of the randomly varying property, is obtained by writing Eq. (10) by

$$
\langle\mathbf{q}\rangle=\varepsilon^{*} \cdot\langle\nabla T\rangle,
$$

where

$$
\varepsilon^{*}=\langle\epsilon\rangle\left[1+\delta_{L}^{*}\right],
$$

$\boldsymbol{\delta}_{L}{ }^{*}$ being obtained from $\boldsymbol{\delta}_{N L}{ }^{*}$ acting on a uniform temperature gradient field of unit strength.

We conclude this section on the Dyson equation by considering the first term on the right-hand side of Eq. (6) in the context of the heat conduction problem. (This is the first 
term to be encountered in which there is a convergence difficulty. Note from Eq. (5) that this term is cancelled in the definition of the effective index operator.) For a homogeneous temperature gradient field and homogeneous statistics (to be achieved in a limit) we can write this term as

$$
\left.\int \mathbf{G}(\mathbf{s}) N_{2}(\mathbf{s}) d \mathbf{s}\right) \cdot\left(\int \mathbf{G}\left(\mathbf{s}_{1}\right) d \mathbf{s}_{1}\right) \cdot\left(\int \mathbf{G}\left(\mathbf{s}_{2}\right) N_{2}\left(\mathbf{s}_{2}\right) d \mathbf{s}_{2}\right)
$$

where $\mathrm{N}_{2}(\mathbf{s})$ is a two-point correlation function defined by

$$
N_{2}(\mathbf{s})=\left\langle\epsilon_{1}(0) \epsilon(\mathbf{s})\right\rangle /\langle\epsilon\rangle^{2} .
$$

It is the interior integral of Eq. (18) that leads to convergence difficulties since $\mathrm{G}(\mathrm{s})$ decays only as fast as $s^{-3}$. Although this integral does not actually diverge in the limit of an unboundedly large region of integration, the value obtained for it depends on the particular order chosen for accomplishing the threefold integration. Thus, if one chooses the infinite domain to be a sphere of unboundedly large radius, one obtains a different value for the integral than for a slab of infinite extent in the limit in which the slab thickness also is allowed to increase without limit. The unsettling aspect of this state of affairs is that possibly the most obvious choice for the outer boundary shape is that of the test specimen [22] leading to a term in the definition of the effective property depending on the specimen geometry. This runs counter, of course, to our intuition and to our experience.

The other three terms of Eq. (6) do not result in a similar difficulty. For example, the second term may be written

$$
\iiint \mathbf{G}\left(\mathrm{u}-\mathrm{s}_{1}-\mathrm{s}_{2}\right) \cdot \mathbf{G}\left(\mathrm{s}_{1}\right) \cdot \mathbf{G}\left(\mathrm{s}_{2}\right) N_{2}(\mathrm{u}) N_{2}\left(\mathrm{~s}_{1}\right) d \mathrm{u} d \mathbf{s}, d \mathrm{~s}_{2} .
$$

The correlation functions fall to zero in a distance that is to be measured on the microscale. Thus, a convergence difficulty might be expected relative to the $s_{2}$ integration. For $s_{2}$ large, however, the integrand of Eq. (20) behaves as $s^{-6}$, which is rapid enough to insure absolute convergence. Similar conclusions can be reached of the third and fourth terms of Eq. (6).

The term of Eq. (6) that is seen to contain the convergence difficulty is readily identified by the topological property that a severing of a solid line segment factors the diagram into two parts. This same rule identifies all conditionally convergent terms arising from the higher-order statistical moments. The series of Eq. (5) shows all such terms to cancel in pairs, leaving as a result a series of absolutely convergent terms. At this stage, then, we are justified in taking the limit in which the region containing fluctuations in $\epsilon(\mathbf{x})$ becomes unboundedly large.

Alternate derivation of bulk property formulae. In this section we accept, a priori, the efficacy of an effective property field theory as given by Eqs. (9) and (16) and address the problem of calculating $\varepsilon^{*}$. No reference will be made to the Dyson equation. Intuitively two definitions of $\varepsilon^{*}$ seem physically reasonable, both of which consider bulk, or averaged, fields, i.e. $\langle\mathbf{q}\rangle$ and $\langle\nabla T\rangle$, which are homogeneous. In one definition we simply average Eq. (8),

$$
\mathrm{q}=\epsilon \mathrm{T},
$$

and set the result equal to $\varepsilon^{*} \cdot\langle\mathbf{T}\rangle$. That is, $\varepsilon^{*}$ is defined by the equation

$$
\varepsilon^{*} \cdot\langle\mathbf{T}\rangle=\langle\epsilon \mathbf{T}\rangle,
$$

where we have used $\mathrm{T}$ to denote the temperature gradient. The second definition of $\varepsilon^{*}$ is 
provided by equating the energy stored, according to the effective index theory, to the averaged energy stored. This leads to the definition of $\varepsilon^{*}$ according to

$$
\langle\mathbf{T}\rangle \cdot \varepsilon^{*} \cdot\langle\mathbf{T}\rangle=\langle\varepsilon \mathbf{T} \cdot \mathbf{T}\rangle \text {. }
$$

It is a well-known and fundamental result of effective property theories that the two definitions of $\varepsilon^{*}$ are equivalent [18]. Here we intend to consider the relationship of the above definition of $\varepsilon^{*}$ to the Dyson equation formalism. We shall do this by using Eqs. (21) and (22) to obtain an iteration (i.e. perturbation) expression for $\varepsilon^{*}$ and compare this expression with that of the last section.

To fix ideas, we consider a large slab-like specimen with a uniform temperature difference maintained over two opposing faces. For the homogeneous averaged slab, the temperature gradient throughout is homogeneous and is given by $G e_{3}$ [23], where $G=$ $\Delta T / h, \Delta T$ being the temperature difference and $h$ being the slab thickness. The unit vector $e_{3}$ is directed normal to the slab faces. A second well-known and fundamental result of effective property theories is that the averaged temperature gradient in the randomly heterogeneous material is also equal to $\mathrm{Ge}_{3}$ [18]. We write

$$
\langle\mathbf{T}\rangle=G \mathbf{e}_{3} .
$$

Thus, to make use of either Eq. (21) or (22) we need only calculate $\langle\epsilon \mathbf{T}\rangle$ or $\langle\epsilon \mathbf{T} \cdot \mathbf{T}\rangle$. It is this calculation that is to be accomplished by an iteration (in our calculation, a perturbation) solution. That is, we perturb the averaged medium solution field, i.e. $G e_{3}$, to obtain a series expansion for $\mathbf{T}$, which may then be used to obtain expressions for $\langle\epsilon \mathbf{T}\rangle$ and $\langle\epsilon \mathbf{T} \cdot \mathbf{T}\rangle$.

In accomplishing the perturbation solution for $\mathrm{T}$ we take the specimen to be of infinite size with a large but finite-sized region containing the variations in $\epsilon(\mathbf{x})$. This makes possible our use of the free-space Green's function in inverting the field variations for the averaged medium. This is valid since a properly formulated prescription for calculating the bulk property will not depend on the size or shape of the specimen. No details of the intermediate calculations are reproduced since they are straightforward, although it is necessary to accomplish an integration by parts at one stage of the calculations in order to obtain results in their most convenient form. The results for $\langle\epsilon \mathrm{T}\rangle$ are written

$$
\langle\epsilon \mathbf{T}\rangle=\langle\epsilon)\left(1+\mathbf{\delta}^{(1)}\right) \cdot G \mathbf{e}_{3}
$$

where the dyad $\delta^{(1)}$ operator can be given a diagrammatic representation, which we write

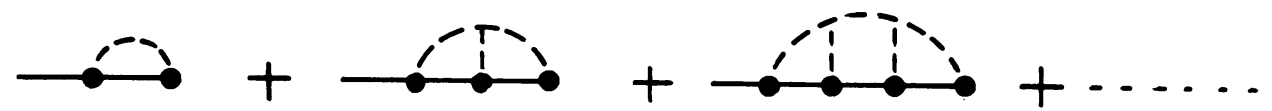

The interpretations to be given to the small dots and the line segments are those of Eqs. (12) and (13). Identifying, now, $\langle\mathbf{T}\rangle$ in the left-hand side of Eq. (21) with $\mathrm{Ge}_{3}$ leads to the following prescription for $\varepsilon^{*}$ :

$$
\varepsilon^{*}=\langle\epsilon\rangle\left[1+\delta^{(1)}\right] .
$$

Thus, this manner of calculating $\varepsilon^{*}$ reintroduces just those conditionally convergent terms which the effective index formulation was designed to remove. In hindsight, this should not be particularly surprising since the heart of the calculation for obtaining $\varepsilon^{*}$ is a calculation of an average of the solution field $\langle\epsilon \mathrm{T}\rangle$, using perturbation theory. Just as an attempt to calculate the averaged field variables $\langle\mathbf{q}\rangle$ and $\langle\nabla T\rangle$ leads to convergence difficulties "requiring the renormalization afforded by the effective index formulation," one should expect to encounter similar difficulties in a similar attempt to calculate $\langle\epsilon \mathbf{T}\rangle$. 
The only troublesome aspect of our calculation is that it appears to throw doubt either on Eq. (21) as a definition, or on Eq. (23) relating the averaged temperature gradient to the boundary conditions applied to the specimen. In fact, both equations are perfectly correct, and it is only the use to which we put them that leads to the difficulty. This is because Eq. (23) can be interpreted as a properly renormalized result for the averaged temperature. Thus, in making use of it in Eq. (21) we have, in effect, renormalized the left-hand side of the equation and not the right-hand side.

The correct prescription for $\varepsilon^{*}$, i.e. that provided by the Dyson equation, can be recovered either by using a nonrenormalized series expression for $\langle\mathbf{T}\rangle$ obtained by averaging the perturbation series for $\mathbf{T}$, or by renormalizing the expression for $\langle\epsilon T\rangle$. In either approach we shall make use of the nonrenormalized series expression for $\langle\mathbf{T}\rangle$ which we write

$$
\langle\mathbf{T}\rangle=\left(1+\delta_{T}\right) \cdot G e_{3}
$$

where $\delta_{T}$ has a diagrammatic representation which we write

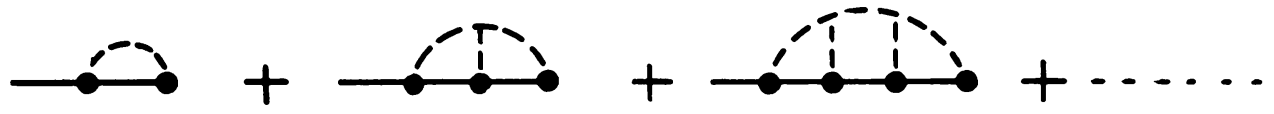

Eqs. (23) and (27) lead to the conclusion that

$$
\boldsymbol{\delta}_{T} \cdot \mathbf{A}=0 \text {, }
$$

for $\mathbf{A}$ being any unitorm vector field. We now consider the nonrenormalized expression for $\langle\epsilon T\rangle$, Eq. (24), and make repeated use of Eq. (29) to write

$$
\begin{aligned}
\langle\epsilon \mathbf{T}\rangle= & \langle\epsilon\rangle\left[1+\boldsymbol{\delta}^{(1)}\right] \cdot G \mathbf{e}_{\boldsymbol{s}} \\
& =\langle\epsilon\rangle\left[1+\boldsymbol{\delta}^{(1)} \cdot\left(1-\boldsymbol{\delta}_{T}+\boldsymbol{\delta}_{T} \cdot \boldsymbol{\delta}_{T}-\cdots\right.\right. \\
& +\underbrace{\boldsymbol{\delta}_{T} \cdot \boldsymbol{\delta}_{T} \cdot \cdots \boldsymbol{\delta}_{T}}_{n \text { times }}+\cdots)] \cdot G \mathbf{e}_{\boldsymbol{s}} .
\end{aligned}
$$

The right-hand side of the second equality of Eq. (30) is a renormalized expression for $\langle\epsilon \mathbf{T}\rangle$. Use of this in Eq. (24) leads to

$$
\varepsilon^{*}=\langle\epsilon\rangle\left[1+\delta^{*}\right]
$$

where

$$
\delta^{*}=\delta^{(1)} \cdot\left(1-\delta_{T}+\delta_{T} \cdot \delta_{T}-\cdots\right) .
$$

On introducing the series representations for $\boldsymbol{\delta}^{(1)}$ and $\boldsymbol{\delta}_{T}$ into the right-hand side of Eq. (31), the result is seen to reproduce term by term the definition of $\delta_{L}{ }^{*}$ obtained from the Dyson equation formalism.

The above "derivation" of a series representation of the bulk property measure would appear to have little to recommend it except that it succeeds. That is, the resulting expression contains none of the conditionally convergent terms that the seemingly more directly derived result, Eq. (26), contains. We note, however, that it is success that appears to provide the justification of all renormalization techniques, such as the Dyson equation formalism that suggests an effective index formulation in the first place.

The definition of $\varepsilon^{*}$ given by Eq. (22) can be considered in a similar, but algebraically 
more complicated, manner as the preceding. The perturbation series for $\mathrm{T}$, when used in $\langle\epsilon \mathbf{T} \cdot \mathbf{T}\rangle$, leads to the result

$$
\langle\epsilon \mathbf{T} \cdot \mathbf{T}\rangle=\langle\epsilon\rangle G^{2} \mathbf{e}_{3} \cdot\left(1+\delta^{(1)}\right) \cdot \mathbf{e}_{3} .
$$

Use of Eqs. (23) and (32) in Eq. (22) gives the incorrect prescription, Eq. (26), for $\varepsilon^{*}$. Upon renormalizing Eq. (32) as above, we obtain the prescription obtained via the Dyson equation formalism.

A comparison of the nonrenormalized and renormalized series expressions for $\varepsilon^{*}$ suggests that one can formally remove the conditionally convergent terms appearing in the former by simply setting them equal to zero. Provided that the description of the statistics of the microstructure is given in terms of cumulants, this formal rule can be interpreted in terms of an operational rule removing all conditionally convergent terms from a computational algorithm for the effective property measure. If the description of the statistics of the microstructure is given in terms other than cumulants, however, the operational rule that is equivalent to the formal rule needs a further explanation. As noted, the conditionally convergent terms of Eq. (5) cancel each other in pairs. Also as noted, the nature of the conditional convergences is that the value of an integral taken over an infinite domain depends on the shape of the outer boundary chosen in specifying the limit process. Thus, if one chooses the outer boundary shape to be such as to make the conditionally convergent terms zero the desired result is achieved. A spherical outer boundary succeeds in this regard. A proper computational algorithm for calculating the effective property measure results from the nonrenormalized series provided one uses a spherical geometry in defining the infinite domain limit. It is of interest that this conclusion is in agreement with the intuitively-argued choice of a spherical geometry as the only reasonable choice for the case of isotropic statistics. We note here, however, that the choice of a spherical geometry is proper even in the event of anisotropic statistics.

We end this section with a discussion of the importance of long-range interactions in determining $\varepsilon^{*}$. It would be erroneous from our calculations to conclude that such interactions are not important in evaluating $\varepsilon^{*}$ to a given order approximation. Thus, for example, a prescription for calculating $\varepsilon^{*}$ for a solid suspension to order $C^{2}$ needs to incorporate long-range interactions in the sense that the two-body calculations needed to incorporate short-range interactions must take note of the fact that the two bodies are located in a suspension and are not isolated in a matrix. The key to taking this notice is Eq. (23). It must be satisfied to order $C^{2}$ if the prescription for evaluating $\varepsilon^{*}$ is to be valid to this order. In the procedure introduced by Batchelor [10], for example, after noting the presence of convergence difficulties as a result of long-range interactions, the renormalization is achieved by subtracting off the conditionally convergent behavior. The conditionally convergent term to be subtracted off is determined by Eq. (23). The similarity between this and Eq. (30) appears clear. In a more recent paper, McCoy and Beran [24] consider a line of reasoning that, while appearing different from that of Batchelor and his colleagues, leads to identical calculations.

Discussion. Even a relatively relaxed review of the literature uncovers numerous examples in which conditionally convergent integrals are tolerated in expressions for effective property measures. Undoubtedly the reason for this is the failure of the investigators to recognize the integrals as such. In those cases in which the integrals were actually evaluated, the investigators arbitrarily assumed an order of integration, seemingly without 
giving the question thought. Fortunately, the most natural order for carrying out the integrations appears to correspond to a spherically symmetric geometry, which as we have seen gives the correct result. In other cases the integrals are never actually evaluated either because the author's interest was in a general formalism or because the integrals appear as part of a parameter that serves to describe the material on the macroscale. In this lastmentioned example, one only needs to reinterpret the parameter in terms of renormalized quantities for the prediction model to be valid.

We cite here just a sampling of studies in which the convergence difficulties were encountered and seemingly ignored. The studies cover the spectrum of cases mentioned above and were chosen either because they appear to be frequently quoted or because they contain results that are, in this author's opinion, significant, or both.

Kröner [25] in an often-quoted paper, in the context of an elasticity problem, uses the analogue of Eq. (21) to define his effective modulus of elasticity tensor series and is led to the nonrenormalized series with a diagrammatic representation given by Eq. (26). He then proceeds to sum the series by introducing the concept of a perfectly disordered material. Perfect disorder is defined by the condition that the material property variations correlate with themselves only for zero separation distance. The statement made is that with this condition all terms such as those termed pairwise clustered terms in referring to Eq. (6) are zero. Hori and Yonezawa [26], in one of a series of papers, criticized Kröner's result in that it does not incorporate what is termed an "exclusion effect." That is, Kröner in effect uses $\left\langle\epsilon_{1}^{4}\right\rangle$ in the term corresponding to the fourth diagram on the right-hand side of Eq. (6) for all points coincident instead of the more proper $\left\langle\epsilon_{1}{ }^{4}\right\rangle-3\left\langle\epsilon_{1}{ }^{2}\right\rangle$. In addition, Hori and Yonezawa argue that the remaining three terms need not be zero, although the first one does calculate out to be so, presumably because of a choice of a spherically symmetric outer boundary in taking the infinite domain limit. Our analysis in this paper demonstrates, therefore, that although the rigor of the analysis in the studies referenced is open to question, the final results cannot be criticized on the grounds of the presence of conditionally convergent integrals.

A nother study of interest from the point of view of the handling of conditionally convergent terms is the study reported by Gubernatis and Krumhansl [7]. These authors actually appear to renormalize in writing a formal solution for the effective property measure. Introducing a completely symbolic equivalence to the various terms of the last section, their Eq. (22) is analogous to our writing

$$
\epsilon^{*}=\langle\epsilon\rangle+\frac{\langle\epsilon\rangle \delta^{(1)}}{1+\delta_{T}},
$$

with the unimportant difference that their base material need not be that determined by the averaging of the material variations. The presence of the term $1+\delta_{T}$ in the denominator appears to arise due to their Eq. (20), which is analogous to our Eq. (27). On inverting the denominator of Eq. (33) and comparing the result to Eq. (30), we see that the formalism of Gubernatis and Krumhansl is identical to that presented herein. An interesting calculation is later performed in which the authors consider the first nontrivial correction to the averaged property measure, which can be taken to represent a zerothorder approximation. The term, given by Eq. (25) of their paper, is of interest since it contains a conditionally convergent component even though only one intermediate "propagation" is involved. We recall from all our previous calculations that the first conditionally convergent term encountered by us required three intermediate "propagations" and represented a third-order correction. The reason why the convergence problem arises 
in the first-order correction term, according to the scheme of Gubernatis and Krumhansl, is that the base material is not that determined by the averaged material. Thus, clusters containing only one point can provide a nonzero contribution. In considering solid suspensions this same reason leads to the presence of conditionally convergent terms in expressions involving only one propagation between inclusions. Of course, the conditionally convergent portion of their term $\langle\delta C G \delta C)$ will be subtracted out by proper use of Eq. (33), i.e. their Eq. (22).

A fourth study of interest is that presented by Elsayed [19] (see also a subsequent paper by Elsayed and McCoy [27]). In Elsayed's treatment, Eq. (22) is taken to define the effective property measure. The right-hand side is then evaluated by writing an approximate expression for $\mathbf{T}$, which is based on a perturbation analysis. Further, the perturbation expression for $\mathrm{T}$ is chosen so as to exactly satisfy a variational formulation of the underlying problem. As a consequence, the approximate expression obtained for $\varepsilon^{*}$ actually represents an exact exact upper bound. A lower bound is obtained by a similar calculation. This procedure is similar to one that had been applied previously by Beran [28], by Beran and Molyneux [29], and by McCoy [30, 31] in obtaining bounds on a variety of property measures. What distinguishes the Elsayed analysis is the order to which he carried out the perturbation analysis. Thus, Elsayed's bounds contain statistical moments up to order five, whereas all of the previously derived bounds contained moments up to order three. The calculations presented in this paper lead one to suspect that Elsayed's bounds will contain conditionally convergent intervals; they in fact do. The calculations also provide a proper interpretation of the bounds; the "packing parameters" that appear in the Elsayed bounds are to be interpreted in terms of renormalized expressions.

Appendix. In this appendix we demonstrate that the effective index operator does not contain conditionally convergent terms. This is most readily accomplished by considering the diagrammatic representation of the generic term for $n$ interactions, represented by a small dot, joined by $(n-1)$ Green's function operators, represented by a solid line. Upon taking the ensemble average and introducing the cumulant expansion, this term is given by a sum of diagrams in which interactions involved in a single cumulant are connected by a dashed line. These diagrams can be collected into two groupings, termed connected and unconnected, with unconnected diagrams defined by the condition that the diagrams can be separated into two, or more, parts by cutting one, or more, solid lines representing the Green's function operator. Thus, for example, four interactions joined by three Green's function operators lead, upon averaging and expanding into cumulants, to four diagrams of which one, i.e.

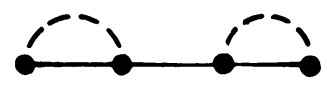

is termed unconnected. Five interactions joined by four Green's function operators lead, upon averaging and expanding into cumulants, to nine diagrams of which two, i.e.

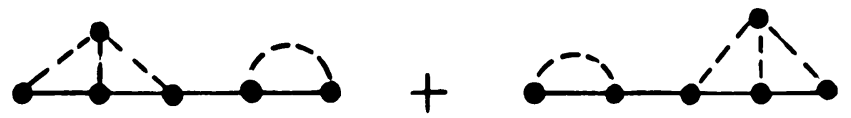

are termed unconnected, etc.

It is the terms represented diagrammatically by the unconnected diagrams that contain conditionally convergent integrals. This is because the volume integrals required by the 
Green's function operator interior to any cluster of interactions joined by a dotted line contain integrands that fall to zero for separation distances that exceed the outer scale correlation length defined on the interactions. The Green's function operator exterior to any cluster of interactions joined by a dotted line, i.e. joining two such clusters, contain integrands that do not fall to zero in a correlation length. The rate of decay of such operators depends only on the rate of fall off of the Green's function itself, that is, as $r^{-3}$ for the unconnected diagrams, which results in the conditional convergence. Connected diagrams of the type, for example,

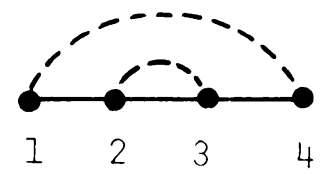

which topologically is equivalent to

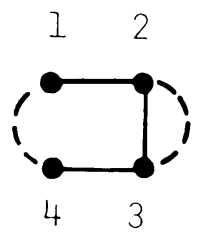

like the unconnected fourth-order diagram, involve two pairs of clustered interactions separated by Green's function operations, but now there is a pair of Green's function operations. Thus, the fall-off rate of the product of interactions is as $r^{-6}$, which doesn't lead to a conditionally convergent integral.

That the effective index operator contains only terms represented diagrammatically by connected Green's function is readily seen by expanding the series definition, introducing the diagrammatic representation, and noting that all unconnected diagrams cancel in pairs. This completes the demonstration.

\section{FOOTNOTES AND REFERENCES}

[1] Statistical homogeneity is obtained in a limit in which the test specimen is unboundedly large.

[2] In an alternate, but equivalent, definition one equates the energy stored in a fictitious, homogeneous effective medium and the averaged energy in the randomly heterogeneous test specimen.

[3] The appropriateness of such an appellation might be questioned by some. I use it here since its use is not uncommon; it is a convenient shorthand term, and the precise meaning is made clear by the equation that describes the formalism.

[4] M. J. Beran and J. J. McCoy, Quart. Appl. Math, 28, 245 (1970)

[5] M. J. Beran and J. J. McCoy, Int. J. Solids Struct. 6, 1035 (1970)

[6] R. Zeller and P. Dederichs, Phys. Stat. Sol. (b)55, 831 (1973)

[7] J. E. Gubernatis and J. A. Krumhansl, J. Math. Phys. 46, 1875 (1975)

[8] G. K. Batchelor and J. T. Green, J. Fluid Mech. 56, 401 (1972)

[9] J. M. Peterson and M. Fixman, J. Chem. Phys. 39, 2516 (1963)

[10] G. K. Batchelor, J. Fluid Mech. 52, 245 (1972)

[11] D. J. Jeffrey, Proc. Roy. Soc. Lond. A335, 355 (1973)

[12] D. J. Jeffrey, Proc. Roy. Soc. Lond. A338, 503 (1974)

[13] R. O’Brien, Ph. D. Dissertation, Cambridge University (1977)

[14] U. Frisch, Probabilistic methods in applied mathematics, ed. A. T. Bharucha-Reid, Academic Press, New York (1968) 
[15] Some authors, including this one, prefer the use of the notation $\langle L\rangle^{-1}$ as more descriptive than $G_{0}$. In the interest of keeping my notation consistent with Frisch [14], I have used the $G_{0}$ notation in this paper.

[16] The outlined proof is clearly only a formal proof. To make the proof rigorous would require a demonstration that all the series involved converge, a task that would have to be accomplished on a problem-byproblem basis. This author knows of no attempt to prove convergence for the effective property problem.

[17] All that has been demonstrated here, of course, is that a proper cancellation is achieved in the term of fourth order in $L_{1}$. In the Appendix we consider terms of higher orders in $L_{1}$.

[18] M. J. Beran, Statistical continuum theories, Interscience Publishers, New York (1968)

[19] M. A. Elsayed, J. Math. Phys. 15, 2001 (1974)

[20] The formalism can be applied to a finite-sized specimen with variations in $\epsilon(\mathbf{x})$ that are "homogeneous" throughout. The effective index operator will exhibit a dependence on the specimen boundary conditions for field points that lie within a neighborhood of the specimen boundary that can be argued to have a thickness that is measured on the microscale. Away from this boundary layer the effective index operator is independent of either the geometry of the boundary surface or the conditions to be satisfied thereon. It is usual to assume that the presence of a boundary layer in the field equations will lead to effects that are similarly localized.

[21] P. Saffman, Studies in Applied Math. 52, 115 (1973)

[22] One might argue that the domain of integration is infinite when viewed on the microscale and that the outer boundary shape is most properly determined by the geometry that can be observed on this scale, i.e. by the statistics of the variations of the property measures. Isotropic statistics can only be compatible with a spherical geometry and, hence, in this case the proper choice for taking the limit is that of a sphere, irrespective of any specimen geometry. While this argument has some appeal since it removes the major source of unease, it is clearly lacking in mathematical rigor.

[23] There is no relationship between $G$ here used to denote the temperature gradient and $G_{0}$ used previously to denote a Green's function operator.

[24] J. J. McCoy and M. J. Beran, Int. J. Engr. Sci. 14, 7 (1976)

[25] E. Kröner, J. Mech. Phys. Solids 15, 319 (1967)

[26] M. Hori and F. Yonezawa, J. Math. Phys. 15, 2177 (1974)

[27] M. A. Elsayed and J. J. McCoy, J. Comp. Mat. 7, 466 (1973)

[28] M. J. Beran, II Nuovo Cimento 38, 771 (1965)

[29] M. J. Beran and J. Molyneux, Quart. Appl. Math. 24, 107 (1966)

[30] J. J. McCoy, Il Nuovo Cimento 57, 139 (1968)

[31] J. J. McCoy, Recent advances in engineering science, Pergamon Press (1970) 